\title{
Alteraciones neuromusculares buco-faciales
}

\author{
Dr. Carlos Pizarro, ${ }^{*}$ Sr. Ficardo Honorato**.
}

\begin{abstract}
ARSTRACT
The present survey shows the results of and interdisciplynary approach to a highly common and neglected problem wich is por oral habits. The sample of 79 cases reveals the hight incidence of the problem in general. The high frecuency of POH detected were buccal breathing (respiration) and atypical deglution.

Fecommendations especially oriented towands their application by especially trained people are suggested and these are geared towards an early identification and derivation of each one of these POH.
\end{abstract}

Las causas o las consecuencias de los malos hábitos orales (MHO) pueden ser motivo de consulta a diferentes profesionales de la Salud, quienes establecen un diagnóstico y tratamiento de acuerdo a su especialidad, descuidando muchas veces el abordaje integral del problema. Un buen ejemplo es el Síndrome del Respirador Bucal, asociado a vegetaciones adenoideas, en el que luego de ser establecida la presencia de tejido hiperplásico se determina como tratamiento su extirpación, sin preocuparse de derivarlo a un Odontólogo-ortodoncista para corregir o descartar la maloclusión, casi siempre presente, $\mathrm{ni}$ al Kinesiólogo para su reeducación miofuncional, sin la cual no se puede considerar completo el tratamiento.

Las secuelas de los malos hábitos orales, en la forma de alteraciones dentomaxilares, faciales, torácicos y posturales son conocidas como para extendernos en su explicación. Nuestra intención es enfatizar que cuando los padres no están lo suficientemente informados, o cuando no existe una adecuada interrelación entre los profesionales in-

\footnotetext{
* Odontología.

"*Kinesiólogo.

Depto. Ciencias de la Salud. Universidad de Chile. Sede

Antofagasta.
}

volucrados en la atención de estos pacientes, los resultados son frecuentemente insatisfactorios. De este modo, es preciso repetir que estos problemas requieren un enfoque multidisciplinario en que cada especialista debe enterarse de lo que puede esperar del otro. Pensamos que lo integral consiste en el enlace entre el Educador, el Pediatra, el Otorrino, el Odontólogo y el Kinesiólogo en la pesquisa y derivación, por una parte, y el oportuno tratamiento ortodóncico y miofuncional como complemento de la atención ORL y cirugía cuando sean necesarias, por otra parte.

Una conducta terapéutica racional debe considerar que la repetición de una conducta anormal de succión, respiración o interposición llega a establecerse en la forma de un PATRON NEURO. MUSCULAR, enfoque que evitara muchos ejercicios inútilmente ensayados y pérdida de tiempo para el enfermo y para el tratante.

Para este estudio hemos elegido niños en edad pre-escolar ( 5 años), por ser ésta una etapa en la que el mal hábito se esta fijando y determinando una serie de trastornos locales y generales.

Durante los primeros años de vida del niño, la enfermera, el pediatra $y$ las educadoras son los únicos profesionales que se relacionan con él. En este sentido recalcamos la importancia de que cualquier mal hábito sea detectado tempranamen- 
te, cuando se está instalando, a fin de establecer un tratamiento precoz.

Normalmente existe un equilibrio entre las fuerzas intra y extra bucales en posición de reposo. A pesar de ser mayor la fuerza intrabucal, representada por la lengua, ${ }^{13}$ se establece un equilibrio en base al efecto hidráulico, elasticidad del carrillo y patrón morfogenético (Weinstein, citado por Graber, ${ }^{5}$ ) cuando se instalan los MHO se rompe este equilibrio y se producen diversos grados de trastomos dentomaxilares. Estudios del rol de la musculatura en la malociusión ${ }^{6}, 13$ electromiografía ${ }^{8,9}$ y su relación con los malos hábitos, son la base del conocimiento actual del tema.

El recién nacido posee un mecanismo bien adaptado para chupar, y su aparato sensible peribucal constituye el medio de intercambio más importante con el medio ambiente..$^{5}$ A través de él no sólo obtiene nutrición, sino también la sensación de bienestar, tan indispensable en las primeras etapas del desarrollo infantil. Anderson' sostiene que los nin̄os amamantados en forma natural están mejor ajustados y poseen menos hábitos musculares peribucales anomales, conservando, tambiên, menos mecanismos infantiles. Si la lactancia se realiza en forma natural o mediante biberones con tetillas anatómicas, $y$ si el niño recibe el cariño y afecto normal de sus padres, la frecuencia de los $\mathrm{MHO}$ se reduce significativamente. ${ }^{5}$ Cuando el niño ha sido alimentado artificialmente, el uso del chupete es quizás necesario para el desarollo de los maxilares. Sin embargo, cuando este hábito persiste más allá de los dos años de edad, es frecuente que se instalen una serie de alteraciones estructurales de los maxilares, tales como compresiones laterales, protrusiones maxilares $y$ mordida abierta anterior. Muchas veces también se retira prematuramente el chupete $y$, como consecuencia de ello, se instala el hábito de chuparse el dedo. Cuando se elige l pulgar, la malformación que se induce es mucho más severa que cuando se elige otro dedo.

Cuando el niño va al colegio por primera vez, cambia el hábito de chuparse el dedo por otro que pase inadvertido, por ejemplo, morderse el labio inferior. Al ubicar el labio inferior detrás de los incisivos superiores, por acción del músculo borla del mentón, se proyecta hacia palatino, aumentando la sobremordida horizontal, y la mandíbula tiende a retruirse. La posición del lảbio inferior da como resultado que al deglutir deje sin acción el labio superior. la lengua se interpone entre los dientes, y así el sellado lingual anterior es reemplazado por un sellado labial. De este modo, la porción posterior de la lengua cae, no realizándose la acción de válvula en la región faríngea ("valvula veloglosa"), constituyéndose el mal hábito de la "deglución atipica". 12

Otro mal hábito que se asocia a malformaciones maxilares es la Respiración Bucal. Este mal hábito puede o no tener como causa directa la obstrucciôn de la vía nasal. El niño cambia su régimen fisiológico nasal, adoptando la vía bucal para respirar en forma permanente o intermitente. Sus secuelas llegan a comprometer a todo el organismo.

Muchas veces las maloclusiones presentes en los niños portadores de malos hábitos tienen una causa hereditaria y se acentúan con estos. También es importante mencionar el hecho de que aquellos niños con oclusión normal (normooclusión, según clasificación de Angle) que presentan MHO tienen una tendencia a normalizar el daño una vez que se establece la dentición definitiva. Pero aquellos niños que establecen una oclusión mesial (clase III de Angle) a distal (clase II de Angle) una vez establecida su dentición definitiva, tienden a magnificar el daño como consecuencía de los $\mathrm{MHO}$, en la medida en que éstos han tenido una mayor duración en el tiempo, una mayor frecuencia durante el día y la noche y una mayor intensidad en su aplicación.

En este trabajo estudiaremos la frecuencia con que aparecen los MHO en pre-escolares de nuestro medio y la relación entre ellos, con el objeto de establecer pautas simples de evaluación que puedan proyectarse hacia un diagnostico inicial que puede ser efectuado por aquellos profesionales que están en contacto con los niños, como las Educadoras de Párvulos. De este modo se puede detectar el problema y efectuar una derivación oportuna.

\section{MATERIAL Y METODO}

Se tomó una muestra de 79 niños de nivel de Transición (5 años de edad) de cinco Jardines Infantiles de la ciudad de Antofagasta, elegido al azar, de acuerdo a la téenica de Muestreo por Conglomerados, aplicando un instrumento, ficha clínica, que permitió determinar los MHO presentes y los signos y síntomas que lo acompañan. En el fichaje. dos aspectos fundamentales: pre- 
guntas a los padres y Educadoras y un examen físico de los encuestados.

Para efectuar el diagnóstico de Respirador Bucal se empleó la prueba de la "mariposa de algodón", que consiste en colocar un algodón en forma de mariposa bajo las tosas nasales. Al hacer inspirar y espirar pennanece inmóvil en la fosa que no funciona cuando hay obstrucción unilateral y cuando no hay respuesta en ninguna de las dos el niño presenta una respiración bucal franca. Además, para confirmar el diagnóstico se investiga con los padres y Educadoras si el niño se fatiga pronto al esfuerzo, y se le efectúa la prueba del espejo, haciéndolo respirar frente a él. Por empanamiento, $o$ ausencia de éste, se efectuará el diagnóstico.

Para diagnosticar una deglución atípica se hace deglutir impidiendo el cierre labial mediante una tracción hacia abajo del labio inferior, colocando el pulgar del exanninador sobre el vestíbulo labial inferior, mientras que con la otra mano se palpa el músculo temporal para sentir la contracción de este músculo, al tiempo que se observa la proyección lingual entre los dientes al momento de tragar.

El diagnóstico de los hábitos de interposición bruxismo se efectuó por observación directa o por descripción de sus padres y educadoras.

\section{ANALISIS DE LOS RESULTADOS}

De los 79 niños examinados, se encontraron 15 sin alteraciones y 64 pottaban algún $\mathrm{MHO}$, lo que corresponde a un $18,9 \%$ en el primer caso y un $81,1 \%$ en el segundo. Como se puede observar en la tabla 1 y figura 1 , la distribución por sexo es la siguiente: Pacientes sin alteraciones, el $40 \%$ son varones y el 60\% mujeres. Entre los portadores de MHO se encontró el $68,4 \%$ de varones y el $31,6 \%$ de mujeres.

La frecuencia de los MHO en relación a los 79 niños examinados es la siguiente: respiradores bucales $48,1 \%$ deglución atipica $41,7 \%, 33$ chupadores de dedos $27,8 \%$, ${ }^{22}$ muerde objetos $16,4 \%,{ }^{13}$ onicotagia $10,1 \%{ }^{+}$finalmente, los bruxómanos constituyeron el $8,8 \%{ }^{7}$

\section{RESPIRACION BUCAL}

Este MHO se presentó en 38 casos, de los cuales, 10 eran puros y el resto se asoció a otros: 6 a deglución atípica; 2 a chupadores de dedos; 1 a
Tabla 1

Mal hábitos orales incidencia-sexus 79 Nin̄os hombres mujeres
N. ${ }^{\circ} \%$ Notal

\begin{tabular}{lllllll}
\hline Niños s/MHO & 6 & 40 & 9 & 60 & 15 & 18,9
\end{tabular}

$\begin{array}{lllllll}\text { Niñus ciMHo } & 3,9 & 68,4 & 25 & 31,6 & 64 & 81,1\end{array}$

\begin{tabular}{lllllll}
\hline Total & 45 & 56,9 & 34 & 43,1 & 79 & 100 \\
\hline
\end{tabular}

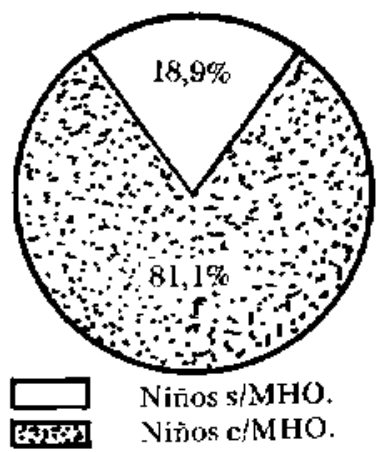

Fig. I. Incidencia general de Ios MHOy su dístribución por sexos.

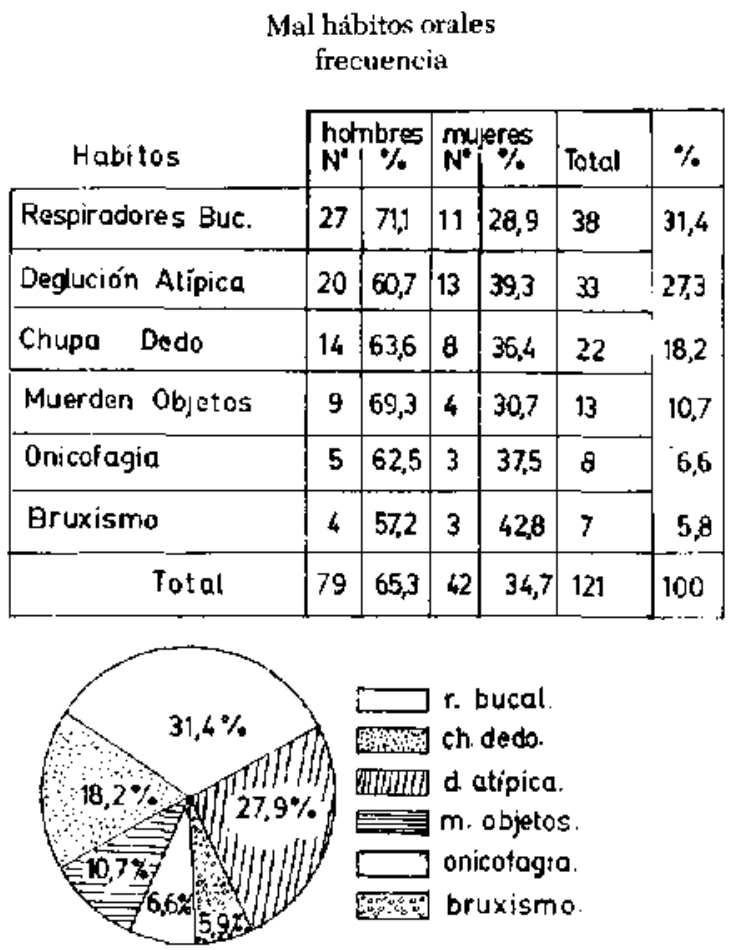

muerdeoljetos; 3 a onicofagia; 4 eran, además de deglutores atípicos, chupadores de dedos; 1 a deglución atípica y muerdeobjetos; 1 a muerdeobje- 
tos y onicofagia; l a deglución atípica y bruxismo; 2 a deglucion atípica, chupadedos y muerdeobjetos; 1 a deglución atipica, chupadedos y bruxismo; 1 a deglución atípica, chupadedos y onicofagia, y 5 a chupadedos y muerde objetos.

Las características, en orden decreciente, de los respiradores bucales fueron las siguientes: el $71 \%{ }^{27}$ usaron biberón; el 47,3\% ${ }^{18}$ usaron chupete; el $42,1 \%$ se resfriaban continuamente; el $39,4 \%$ presentaban amigdalas hipertróticas; el $31,5 \%^{12}$ con mordida abierta anterior; el $26,3 \% \%^{9}$ tenían pie plano; el $21 \%^{8}$ respiración costal superior; el $18,4 \%{ }^{7}$ presentaban facies adenoideas; el $13 \% 5$ paladar alto; el $13 \%{ }^{5}$ labio superior corto; el $13 \%$ compresión lateral maxilar superior y el $10 \%{ }^{4}$ presentaban tórax estrecho.

\section{DEGLUCION ATIPICA}

Este mal hábito se presentó en 33 casos, de los cuales ' 12 eran puros y el resto estaba asociado a otros MHO: 6 con respiración bucal; 4 chupadores de dedos; 4 con respiración bucal y chupadores de dedos; 1 con respiración bucal y onicofagia; 1 con respiración bucal y muerdeobjetos; 2 con respiración bucal, chupadores de dedos y muerdeobjetos; y l con respiración bucal, chupadedos y bruxismo.

Las características del $\mathrm{MHO}$ en orden decreciente fueron las sigujentes: uso de biberón $57 \%$; ${ }^{19}$ uso de chupete $48 \%{ }^{16}$ amígdalas hipertroficas $45 \%$; ${ }^{15}$ mordida abierta anterior $39 \% ;^{13}$ chupan dedos $30 \% ;^{10}$ protrusión maxilar $18 \%{ }^{; 5}$ compresión maxilar $15 \% ;{ }^{5}$ labio superior corto $12 \% ;{ }^{4}$ engua descendida $12 \% ;^{4}$ y paladar alto $12 \% .^{4}$

\section{CHUPADORES DE DEDOS}

El MHO de chuparse los dedos se presentó en forma pura en 2 casos del total de 22 . El resto se asocí a los siguientes malos hábitos: 2 a respiración bucal; 4 a deglución atípica; 1 a morder objetos; 4 a respiración bucal y deglución atipica; 5 a respiración bucal y muerdeobjetos; 2 a respiración bucal, deglución atípica y muerdeobjetos; 1 a respiración bucal, deglución atipica y bruxismo; y 1 a respiración bucal, deglución atípica y onicofagia.

Las características de este mal hábito, en orden decreciente, fueron las siguientes: uso de biberón $68 \% ;^{15}$ mordida abierta anterior $59 \%,{ }^{13}$ uso de chupete $50 \%{ }^{11}$ muerden objetos $40 \% \%^{9}$ diastemas
$31 \% ;^{7}$ paladar alto $13 \% ;^{3}$ y compresión maxilar $9 \%{ }^{2}$

\section{MUERDEOBJETOS}

Corresponden a 13 casos en total, de los cuales solamente un caso se presentó en forma pura. El resto se asoció a otro en la siguiente proporción: I a respiración bucal; 1 a chupadedo; 1 a a-bruxismo; 5 a respiración bucal $y$ chupadedo; 1 a respiración bucal y deglución atípica; 1 a respiración bucal $y$ bruxismo; 1 a chupadedo y onicofagia; 2 a respiración bucal, deglución atípica y chupadedo; $y$, finalmente, 1 a respiración bucal, deglución atípica y onicofagia.

\section{ONICOFAGIA}

Se presentó en ocho casos (8), de los cuales en sólo 2 se observó en forma pura, y el resto se asoció a otros malos hábitos: 3 a respiración bucal; 1 a respiración bucal y deglución atípica; 1 a chupadedo y muerde objetos; y 1 a respiración bucal deglución atípica y chupadedo.

\section{BHUXISMO}

Lo encontramos en 7 oportunidades, de las cuales 2 eran puros y el resto se asoció a: deglución atípica 1; muercleobjetos 1 ; respiración bucal y deglución atípica 1 ; respiración bucal y muerde objeto 1 ; y a respiración bucal, deglución atípica y chupadedo 1 .

\section{CONCLUSIONES}

1. Se observa una alta incidencia de $\mathrm{MHO}$ en preescolares.

2. Los MHO predominan en el sexo masculino.

3. La asociación de dos o más MHO es lo habitual.

4. La respiración bucal y la deglución atípica son los MHO predominantes.

5. El uso prolongado de biberón y chupete, resfrio frecuente e hipertrofia amigdaliana son las características que se observan con mayor frecuencia.

6. Las secuelas predominantes fueron: mordida abierta anterior, compresión lateral maxilar y paladar alto.

7. El respirador bucal presentó además las si- 
guientes secuelas: pie plano, respiración costal superior y tórax estrecho.

8. El deglutor atípico presentó, además de las secuelas enumeradas en la conclusión 6, labio superior corto y lengua descendida.

9. Los MHO de interposición (chupadedo y muerdeobjeto) presentaron como secuelas diastemas y maloclusión focal.

\section{RECOMENDACIONES}

1. Respiración bucal. Al constatar ronquido nocturno, boca entreabierta persistente, fatiga precoz al esfuerzo físico en niños con antecedentes de resfrío continuo y/o anigdalitis repetidas, debe sospecharse de un mal hábito de respiración bucal. La conducta a seguir debe ser la siguiente:

a) Derivación al Otorrino para descartar o confirmar obstrucción de la vía aérea superior.

b) Derivación al Odontólogo para determinar magnitud de tas seeuelas dentomaxilo-facinles; $y$ procedencia de un tratamiento ortodóncico.

c) Derivación al Kinesiólogo para el tratamiento miofacial correspondiente.

2. Deglución atipica. Al constatar la presencia de la lengua interpuesta constantemente entre los dientes, pronunciación defectuosa con ceceo, y lengua descendida en niños con antecedentes de uso prolongado de biberón o chupete, debe sospecharse hábito de deglución atípica. La conducta debe ser la siguiente:

a) Derivación al Odontólogo para establecer el diagnóstico y magnitud de las alteraciones secuenciales.

b) Derivación al Kinesiólogo para programar el tratamiento miofuncional.

c) Derivación al Fonoaudiologo en caso de persistir la dificultad en la articulación de algunos fonemas.

3. Hábitos de interposición. Al constatar que el níno chupa alguno de los dedos de la mano, muerde lápices u otros objetos, usa biberón o chupete después de los dos años y medio de edad o se interpone el labio inferior o la lengua entre los dientes, se está confirmando el mal hábito de in- terposición. Debe derivarse a los mismos profesionales mencionados a propósito de la deglución atípica.

Los padres y educadores deben adoptar una conducta sjcológicamente adecuada al colaborar en la eliminación de estos hábitos de interposición, evitando entrar en "competencia" con el niño. Cuando la corrección es muy vehemente el niño reacciona negativamente, acentuando el mal hábito.

\section{RESUMEN}

Este estudio comunica los resultados de un abordaje interdisciplinario de un problema tan común y tan descuidado como los malos hábitos orales. La muestra de 79 casos refleja la alta incidencia de este problema en general. Los dos MHO más frecuentes fueron respiración bucal y deglución atípica. Se dan recomendaciones, especialmente orientadas a su aplicación, por educadoras enfocadas al reconocimiento y derivación oportuna de cada uno de estos MHO.

\section{REFERENCIAS}

IAnderson, $W$. The relationship of the tongue-thrust Syndrome to naturation and other factors. Am. J. Orthodont. 40: 264-275. 1963.

${ }^{2}$ Ferrer, C. y Col. El niño respirador bueal. Rev, Otorrino. 36 $59-65,1976$

${ }^{3}$ Gesell, A. and Ifg, F. Intant and child in the culture of today, 2 Ed. Philadelphia H. and B. 1958.

${ }^{4}$ Cruber, T.M. the three $M$ 's: Muscles, malfortoation and mal oclusion Am. J. Othodoncic 49: 418-450, 1963.

${ }^{5}$ Graber, T.M. Ortodoncia $3 \mathrm{Ed}$. M exico, Interamericana. $19 \overline{7} 4$.

${ }^{6}$ Hotz, $\boldsymbol{k}$. Orthodontia in every day Practice. Beme, Hans Huber, 1971 .

${ }^{7}$ Lear, $C$. et al. The frecuency of deglution in man. Arch Oral Biolngy 10: 83-93, 1965.

${ }^{8}$ Moyers, R.E. Handbook of Orthodontic. 3 Ed. Chicago Year book Pub., 1972.

9Moyers, R.E. Fol de la lengua en has maloclusiones. Odont. Cliniça de Yorte América. 23: 325 . Interamericana, 1968.

10 Orellana, C.: Respirador Bucal. Tesis para optar titulo Kine. síblogo, 1967

I1 Rugers, $A_{-}$and Other: Symposium on muscle function. Irternat. J. Orthodont. 16: 254.1930.

12Wildman, A.: Aparato motor. Am. Dent. Clinic. 20: 69l-705, Oet. 1976.

13Winder, R.: An electronic technique to measure the forces exerted on the dentition by the dentition perioral and lingual musculature. An. Dent. J. Orthodontic 42: 645, 1956. 